\title{
Influence of Power Control Strategies on the Voltage Profile in an Islanded Microgrid
}

\author{
T.L. Vandoorn ${ }^{1}$, T. Loix ${ }^{2}$, L. Vandevelde ${ }^{1}$, B. Meersman ${ }^{1}$ and J. Driesen ${ }^{2}$ \\ ${ }^{1}$ Electrical Energy Laboratory (EELAB), Department of Electrical Energy, Systems \& Automation, \\ Ghent University, Sint-Pietersnieuwstraat 41,9000 Gent, Belgium, Tine.Vandoorn@UGent.be \\ ${ }^{2}$ K.U.Leuven ESAT/ELECTA \\ Kasteelpark Arenberg 10, 3001 Leuven, Belgium
}

\begin{abstract}
The emergence of large amounts of distributed energy resources (DER) poses new challenges for the active participation of those units in the control of the grid. In this context, a recent development is the microgrid, which presents a coordinated approach for integrating the DER in the electrical grid. Microgrids are able to operate either connected to the distribution grid or in islanded mode. In this paper, the influence of different power control strategies of DER is studied with respect to the voltage profile in an islanded microgrid. A description of the control strategies is presented, as well as some simulation results for a basic microgrid.

Index Terms-Microgrids, distributed generation, voltage con-
\end{abstract} trol, voltage-source inverter

\section{INTRODUCTION}

The number of distributed energy resources (DER), with often a primary energy source of renewable origin, increases steadily since the last two decades. A significant share of the DER uses a power electronic converter for coupling the unit to the grid. The impact of these units on the quality of the grid supply and their optimal integration has been studied by many authors [1]-[3]. New grid topologies have been proposed to facilitate the integration of DER and minimize any negative impact on the grid and the customers connected to it, amongst which the microgrid has gained considerable popularity [4]. Although the microgrid topology offers several advantages from the point of view of load-supply balancing, reliability of supply and possibly power quality, it also has some weaknesses and issues that must be solved before it can be implemented on a large scale [5], [6]. One of the issues is the voltage profile in the microgrid. This paper describes this aspect and shows that inverter-connected sources can alleviate the problem, given an adequate control algorithm.

Therefore, in this paper, the voltage profile in a small isolated microgrid is studied for different power control strategies. The first control strategy allows certain voltage deviations in the microgrid, while the other strategies adjust their generated power to reduce the voltage deviations. Two approaches for this are presented here: a first control strategy adjusts its generated power without communication with the other generators, and the second one is based on a gossipingbased communication strategy to minimize the average voltage deviation.

\section{MicrogRid ACTIVE POWER CONTROLLERS}

The active power controllers in the microgrid need to take into account the specific characteristics of the microgrid.

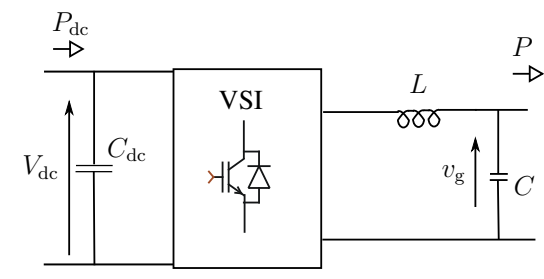

Fig. 1. Power source with power-electronic interface to the microgrid

First, opposed to conventional power systems, in islanded microgrids, there is a lack of inertia as most grid elements are power-electronically interfaced. Secondly, unlike the inductive transmission systems, here, the microgrid has resistive lines like the cabled low-voltage networks. Furthermore, for reliability issues, the usage of a communication link for the primary control is avoided.

The microgrid elements are mainly interfaced to the grid via voltage-source inverters (VSIs). The VSI-interface of the studied microgrid configuration is depicted in Fig. 1. The acside of the VSI is connected to the microgrid via an LC filter to attenuate switching ripple. The dc-side is connected to a power source via a dc-bus capacitor $C_{\mathrm{dc}}$.

All control structures in this paper aim to obtain a maximally reliable system and a fully distributed operation, avoiding single-point of failures such as master units or central controllers. Therefore, the following control strategies for the grid control are studied. Firstly, a combination of droop control strategies is presented, with $P / V$-droop control and $V_{\mathrm{g}} / V_{\mathrm{dc}^{-}}$ droop control of [7], [8] for the active power control, combined with $Q / f$-droop control for the reactive power control [9]. Secondly, a power control strategy based on primary and secondary control actions is presented [10]. The primary control is achieved by $P / V$-droop control and the secondary control action is obtained with a gossiping technique.

\section{A. $V_{\mathrm{g}} / V_{\mathrm{dc}}$-droop controller}

For the active power control in the islanded microgrid, firstly, the $V_{\mathrm{g}} / V_{\mathrm{dc}}$-droop control strategy is applied, with $V_{\mathrm{g}}$ the rms value of the grid voltage and $V_{\mathrm{dc}}$ the dc-bus voltage. For each VSI in the microgrid, the dc-bus voltage enables comparing the generated dc-power $P_{\mathrm{dc}}$ with the ac-power $P$ that is delivered to the microgrid. For example, an increasing $V_{\mathrm{dc}}$ indicates an excess of generated dc-power. Therefore, the rms grid voltage $V_{\mathrm{g}}$ is adapted with the dc-bus voltage $V_{\mathrm{dc}}$, 
with a positive droop, and the balancing is achieved by using the resistive nature of the microgrid loads and lines.

The $V_{\mathrm{g}} / V_{\mathrm{dc}}$-droop controller uses the limited transient power storage capabilities of the dc-bus capacitor while avoiding frequent changes of the generated power. Furthermore, also the specific microgrid properties are taken into account. Firstly, inertia is not necessary for the operation of the power controller. Secondly, the droop with $V_{\mathrm{g}}$ arises from the linkage between the active power and the grid voltage rms value, caused by the resistive nature of the microgrid lines. Thirdly, with this control strategy, the dc-power remains constant, and therefore, uncontrollable power sources can operate longer in a nominal condition. Also, the power balancing is achieved without communication between the generators.

\section{B. $P_{\mathrm{dc}} / V_{\mathrm{g}}$-droop controller}

Generally, in the microgrid, the $V_{\mathrm{g}} / V_{\mathrm{dc}}$-droop controller can not be used solely, as in this case, the power flexibility lies only in the dc-bus capacitors and the grid voltage. Therefore, also a $P_{\mathrm{dc}} / V_{\mathrm{g}}$-droop controller is suggested to include flexibility in the generated power. As in resistive lines, a strong linkage between the active power and the rms voltage is present, this controller droops the generated dc-power $P_{\mathrm{dc}}$ with the grid voltage $V_{\mathrm{g}}$, with a negative droop slope. In this way, the generated power is changed according to the voltage level. Furthermore, the droop can be adjusted to control the contribution in power sharing of the power sources. This is analogous to the primary control in the conventional system with an active power / frequency droop.

\section{Combination of $V_{\mathrm{g}} / V_{\mathrm{dc}}$ and $P_{\mathrm{dc}} / V_{\mathrm{g}}$-droop controller}

Also, the $V_{\mathrm{g}} / V_{\mathrm{dc}}$ and $P_{\mathrm{dc}} / V_{\mathrm{g}}$-droop control strategies can be combined. In a certain voltage band, the constant-power band in Fig. 2, only $V_{\mathrm{g}} / V_{\mathrm{dc}}$-droop control is used, with a constant output power. In case the voltage exceeds this voltage band, also the $P_{\mathrm{dc}} / V_{\mathrm{g}}$-droop controller is put into service. The combination of these control strategies is proposed to exploit the advantages of both. The $V_{\mathrm{g}} / V_{\mathrm{dc}}$-droop control strategy is interesting for its constant output power, avoiding frequent changes of the generated power. The latter is specifically interesting for renewables in optimizing their output power. Also, in microgrids, a tolerated voltage deviation from its nominal value is allowed [11], and the $V_{\mathrm{g}} / V_{\mathrm{dc}}$-droop control strategy uses this effectively. However, still, some limits are imposed. Therefore, a voltage limiting procedure needs to be included, which is delivered by the $P_{\mathrm{dc}} / V_{\mathrm{g}}$-droop controller that changes the generated power in case the voltage leaves the constant-power band.

The width of the constant-power band can be adjusted to meet the specific characteristics of the sources. Consequently, by setting the constant-power band, a distinction in the power sharing involvement of the controllable sources and the uncontrollable ones can be made. For example, slightly controllable or uncontrollable (intermittent) power sources have a wide constant-power band and, in this way, $P_{\mathrm{dc}}$ is left unchanged during larger variations of $V_{\mathrm{g}}$. On the other hand, controllable power sources, which can change their fuel intake fast and easily or use storage, have a smaller constant-power band, and therefore, they take a larger part in the power control of the

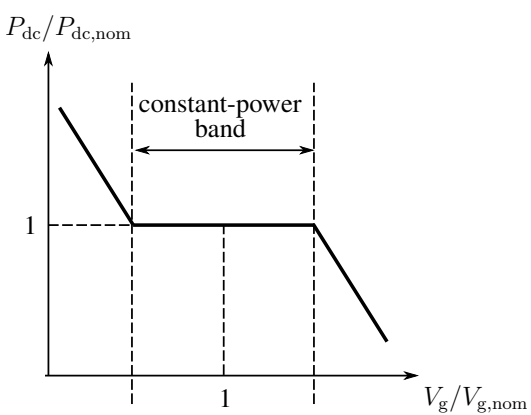

Fig. 2. $\quad V_{\mathrm{g}} / V_{\mathrm{dc}}$ and $P_{\mathrm{dc}} / V_{\mathrm{g}}$-droop controller: constant power-band

microgrid.

In conclusion, the $V_{\mathrm{g}} / V_{\mathrm{dc}}$-droop controller exploits the tolerable voltage deviation to avoid frequent changes in the delivered ac-power. This is advantageous for uncontrollable sources (e.g., some renewables operating in an optimal condition) in order to use the (renewable) energy more effectively. The $P_{\mathrm{dc}} / V_{\mathrm{g}}$-droop controller on the other hand, helps restricting the grid voltage by changing the generated power or by using storage facilities. Furthermore, also other elements can operate with the same control strategies. For example, storage elements can adjust their power consumption or delivery analogously to the $P_{\mathrm{dc}} / V_{\mathrm{g}}$-droop controller of the generators.

\section{Gossip-based secondary control}

The fourth control strategy consists of a primary droop control and a secondary gossiping-based control.

The purpose of primary control is to stop the frequency moving away from its nominal value. This is achieved using droop control: the output power of the inverter is changed proportionally to the frequency deviation. The same principle is applied for the local voltage magnitude control. In a transmission grid with inertia and predominantly inductive line impedance, the active power is used to influence the frequency and the reactive power exchange controls the local voltage magnitude. In case of a predominantly resistive line impedance, this linkage is reversed: the active power influences the local voltage magnitude, while the reactive power controls the frequency or phase-angle.

The droop control principles are implemented by using virtual output impedance emulation. The system emulated by the inverter is depicted in Fig. 3(a) and consists of a voltage source $u_{\text {src }}$ with a series output impedance $Z_{d}=R_{d}+j X_{d}$. The current source $i_{\text {src }}$ is used to model active and/or reactive offset power exchanged between the inverter and the grid. The actual physical inverter system is shown in Fig. 3(b), using an LCL output filter for interfacing the inverter to the grid. Details concerning the correspondence between droop control and virtual output impedance emulation are described in [12] and [13]. One of the main advantages of using the virtual output impedance emulation technique is that harmonics are automatically taken into account, which is not the case for traditional droop control. The latter finds its origin in the control of central synchronous generators connected to the transmission network, in which harmonics are generally not an issue. 


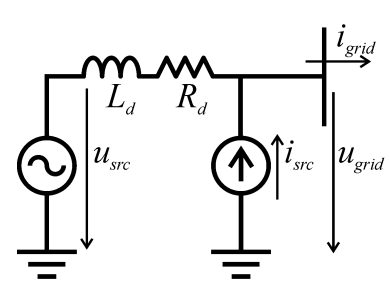

(a)

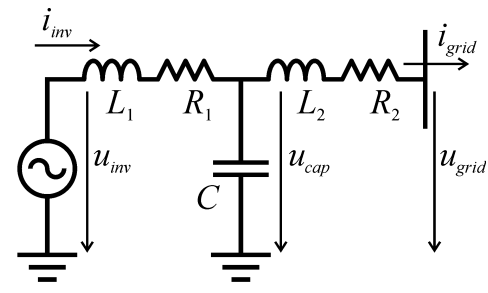

(b)
Fig. 3. (a) Emulated system model, (b) Actual inverter set-up

Traditional power system control includes multiple control levels [14]. After an unbalance occurs between the generated and consumed active power, the primary frequency control limits the initial frequency deviation. The purpose of the secondary control is to regulate the frequency back to its nominal value. This principle is currently used in the European interconnected grid, but can also be applied to smaller systems, such as microgrids [10]. Therefore, in [13], primary control is achieved using a voltage and frequency droop control algorithm that does not require any inter-unit communication, as its operation depends on locally available data. The secondary control involves communication between the various inverters and is implemented by using the gossiping technique [15]. Gossiping is a technique for quickly disseminating and aggregating data in a large (distributed) network or obtaining global system information at local nodes in the network without central coordination. The basic principle is that each network node periodically and randomly selects a neighbour with which it exchanges data. Each node adjusts its local value using a specified update function operating on the exchanged data, for instance, averaging the values of both nodes. The gossiping-based secondary control must minimize the average of all voltage and frequency deviations, as measured locally by the distributed generation (DG) units. Whereas frequency deviations are the same at all DG units, voltage deviations differ depending on the local load and supply and the line impedances.

First, the primary control limits the voltage and frequency excursions following a power unbalance in the system by introducing control actions $P_{\text {prim }}$ and $Q_{\text {prim }}$ at each DG unit. Then, the secondary control achieves minimal voltage and frequency deviations by ensuring that the average of the deviations $P_{\text {prim }}$ and $Q_{\text {prim }}$ is brought to zero. The secondary control actions replace those of the primary control, resulting in improved power quality. The average values $P_{\mathrm{sec}}$ and $Q_{\mathrm{sec}}$ of the primary control actions $P_{\text {prim }}$ and $Q_{\text {prim }}$ for units 1 and 2 are calculated as:

$$
\begin{aligned}
P_{\text {sec }}[k] & =\frac{\bar{P}_{\text {prim }, 1}[k]+\bar{P}_{\text {prim }, 2}[k]}{2} \\
Q_{\text {sec }}[k] & =\frac{\bar{Q}_{\text {prim }, 1}[k]+\bar{Q}_{\text {prim }, 2}[k]}{2}
\end{aligned}
$$

The average values of the active and reactive primary control actions are given by:

$$
\begin{gathered}
\bar{P}_{\text {prim }}[k]=P_{\text {sec }}[k-1]+P_{\text {prim }}[k]-P_{\text {prim }}[k-1] \\
\bar{Q}_{\text {prim }}[k]=Q_{\text {sec }}[k-1]+Q_{\text {prim }}[k]-Q_{\text {prim }}[k-1]
\end{gathered}
$$

The secondary control actions $P_{\mathrm{sec}}$ and $Q_{\mathrm{sec}}$ are added to the active and reactive power references for the primary control block periodically. In this case, the power set points are updated after four gossiping time steps. In this way, a trade-off between fast updating, thus, a fast secondary control, and high accuracy is obtained. This structure is in fact a distributed PI controller, adapting the $P_{\mathrm{sec}}$ and $Q_{\mathrm{sec}}$ of each unit in such a way that the system converges to a situation where the sum of the power deviations $P_{\text {prim }}$ and $Q_{\text {prim }}$ are both zero. As a result, the steady-state voltage and frequency deviations are located within their tolerated zones, compliant with the grid code. Only in the presence of long lines with large loads or distant energy sources, the voltage deviation may get outside of the tolerated zone, as in this case it is impossible to obtain voltage deviations within the allowed range at all locations in the grid. The algorithm ensures that the sum of the excess voltage deviations outside the tolerated zone is minimal.

\section{E. Conclusions}

Different control strategies for adjusting the voltage profile in islanded microgrids are presented. In the first control strategy, the $V_{\mathrm{g}} / V_{\mathrm{dc}}$-droop control strategy, $V_{\mathrm{g}}$ is effectively used for balancing the power in the islanded microgrid while no restrictions on the grid voltage are made. In the $P_{\mathrm{dc}} / V_{\mathrm{g}}-$ droop control strategy on the other hand, the sources adjust their power automatically and without communication to force the grid voltage closer to its nominal value. The third controller uses a combination of the aforementioned control strategies to differ the contribution of the generators. In this way, some generators take a larger part in the power control and voltage profile regulation, while others avoid frequent power changes and optimize their (renewable) output power. The fourth control strategy uses a secondary gossiping-based control algorithm to adjust the voltage profile in the microgrid. After the gossiping procedure, generally, the voltage is forced in a tolerated voltage band.

\section{CASE STUDY: DROOP CONTROLLERS WITHOUT COMMUNICATION}

For studying the voltage profile that is obtained by the different control strategies, the basic microgrid configuration of Fig. 4 is suggested. It consists of two VSI-interfaced power sources feeding two resistive loads via line resistances. The power sources have equal ratings, with $P_{1, \text { nom }}=P_{2, \text { nom }}=$ $3 \mathrm{~kW}$. Load 1 equals $200 \Omega$ and load 2 is a larger load of $10 \Omega$. As shown in Fig. 1, both inverters have an LC filter at the ac-side for attenuating switching ripple, with $L=2 \mathrm{mH}$ and $C=3 \mu \mathrm{F}$, and the power source is connected to the VSI via a dc-bus capacitor $C_{\mathrm{dc}}=1.5 \mathrm{mF}$. The nominal dc-bus voltages equal $450 \mathrm{~V}$. For the reactive power controllers, the droops of both sources are equal to $0.05 \mathrm{mHz} / \mathrm{VAr}$. For the $P_{\mathrm{dc}} / V_{\mathrm{g}}$-droop controllers, the droops equal $-10 \mathrm{~W} / \mathrm{V}$, unless it is stated otherwise. The simulations, including the active power controllers, start from $230 \mathrm{~V} \mathrm{rms}$ at $\mathrm{t}=0 \mathrm{~s}$. The reactive power controllers start operating after $100 \mathrm{~ms}$.

As stated above, the load near VSI 2 is very high compared to the one near VSI 1. This strongly influences the voltage profile and shows an example of a difficult scenario for the control of the microgrid voltage profile. The droop controllers 


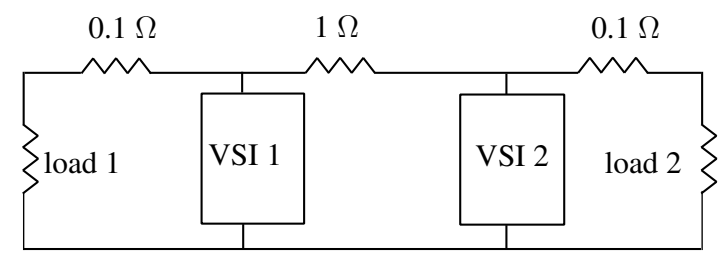

Fig. 4. Basic microgrid configuration, with two DG units (with VSI-interface) and two loads

change the voltage profile without communication between the generators. The simulations are divided into three cases:

- Case I: $V_{\mathrm{g}} / V_{\mathrm{dc}}$-droop controller

In this simulation case, the dc-powers of the sources remain constant according to §II-A. Therefore, the power balancing is provided by the loads and possible energy storage elements only.

- Case II: $V_{\mathrm{g}} / V_{\mathrm{dc}}$ and $P_{\mathrm{dc}} / V_{\mathrm{g}}$-droop controller By the combination of these droop control strategies, the output power of the DG units remains constant in a constant-power band, while if a certain voltage level is exceeded, the delivered ac-power changes. Four subcases are studied.

In sub-case IIa, this voltage level is set to $5 \%$ deviation of the nominal voltage according to §II-C. In this way, a slightly-controllable generator can be emulated. In subcase IIb, the deviation is $0 \%$, so only the $P_{\mathrm{dc}} / V_{\mathrm{g}}$-droop controller is used in a fully-controllable power source according to $\S \mathrm{II}-\mathrm{B}$. An advantage of the control strategy presented is that the slightly-controllable DG units can operate at constant power during a larger amount of time, while the control abilities of the controllable generators are fully exploited.

In the last two sub-cases, the effect of the droop on the voltage profile is studied. In sub-case IIc, it is shown that by increasing the absolute value of the slope, the generator can take a larger part in the power sharing, forcing the grid voltage closer to its nominal value. However, as shown in sub-case IId, this increase is limited by the nervousness of the controller, including stability margins.

The combination of the $V_{\mathrm{g}} / V_{\mathrm{dc}}$-droop controller and the $P_{\mathrm{dc}} / V_{\mathrm{g}}$-droop controller assures a good power sharing and balancing, with limited voltage deviations, while using the tolerable voltage band effectively.

- Case III: Constant terminal voltage

In this case, the controllers adjust their output powers in order to keep their terminal voltages constant. This is a limit case for studying the steady-state behavior of (secondary) controllers that force $V_{\mathrm{g}}$ strictly to its nominal value without restriction of the generated power. Because of the chosen asymmetrical microgrid configuration, with very different loads, in this case, the generators suffer from very high power variations. Furthermore, these high power changes can be avoided by the aforementioned droop control strategies that properly use the allowance of the grid voltage to deviate from its nominal value.

\section{A. Case I: $V_{\mathrm{g}} / V_{\mathrm{dc}}$-droop controller}

In this simulation case, the delivered active powers of both sources remain constant. By using the $V_{\mathrm{g}} / V_{\mathrm{dc}}$-droop controller, the dc-bus voltage is used as a measure to adjust the grid voltage for the power balancing in the microgrid.

The simulation results are depicted in Fig. 5. Note that power measurements are only valid after a fundamental period, hence the initial value of zero. In Fig. 5(c), it is shown that initially, the dc-bus voltage increases because the delivered active power is lower than the generated power of $3 \mathrm{~kW}$. Also, after start-up, the voltage $V_{\mathrm{dc}, 2}$ increases slower than that of VSI 1, as near VSI 2 the highest load (i.e. lowest resistance) is present. With increasing dc-bus voltage, the $V_{\mathrm{g}} / V_{\mathrm{dc}}$-droop controller will increase the set point of $V_{\mathrm{g}}$ as depicted in Fig. 5(a). This process goes on until $V_{\mathrm{g}}$ and $P$ remain constant. In steady-state, both VSIs deliver the same power of $3 \mathrm{~kW}$, with $V_{\mathrm{g}, 1}$ larger than $V_{\mathrm{g}, 2}$. The reason is that the load near VSI 1 is lower. Also, from $V_{\mathrm{g}, 1}$ larger than $V_{\mathrm{g}, 2}$, it follows that $V_{\mathrm{dc}, 1}$ is higher than $V_{\mathrm{dc}, 2}$ because both controllers have equal, positive slopes.

For the reactive power controllers, initially $Q_{1}$ is lower than $Q_{2}$, mainly because the resistance near VSI 1 is higher than that near VSI 2. Therefore, the $Q / f$-droop controllers make $f_{2}$ temporarily higher than $f_{1}$, thus increasing the phase angle of VSI 2 with respect to VSI 1. The voltage of VSI 2 will increasingly lead that of VSI 1 , lowering $Q_{2}$, while increasing $Q_{1}$. This process goes on until in steady-state $f_{1}=f_{2}$ and $Q_{1}=Q_{2}$. As the reactive power is measured after the LC filter, no inductive or capacitive grid elements are present in the simulated microgrid and, thus, in steady-state, $Q_{1}=Q_{2}=0 \mathrm{VAr}$ and $f_{1}=f_{2}=50 \mathrm{~Hz}$.

The voltage profile is depicted in Fig. 5(f). In the case with constant delivered powers, the voltage can vary significantly if the microgrid configuration is very asymmetrical. Also, the deviations of the grid voltage from the nominal voltage are determined by the delivered power and the microgrid configuration, while no control of this is included. The results are summarized in Table I.

It is concluded that with the $V_{\mathrm{g}} / V_{\mathrm{dc}}$-droop controller only and, thus, constant delivered active powers, the voltage can vary significantly in the microgrid. This voltage variation depends on the microgrid configuration. Also, no voltage limiting is incorporated in the microgrid. Still, the $V_{\mathrm{g}} / V_{\mathrm{dc}^{-}}$ droop controller can be very advantageous for uncontrollable power sources. It exploits a tolerated grid voltage deviation effectively, leading to less variations in the generated power of the power sources. The usage of this voltage deviation is thus very advantageous. However, more flexibility in the microgrid is required, certainly in small isolated microgrids where the load peak can be very high. Therefore, also the delivered power of the sources needs to vary, which is the case in the following simulations.

\section{B. Case II: $V_{\mathrm{g}} / V_{\mathrm{dc}}$ and $P_{\mathrm{dc}} / N_{\mathrm{g}}$-droop controller}

\section{1) Case IIa: $5 \%$ constant-power band}

In this simulation, the $P_{\mathrm{dc}} / V_{\mathrm{g}}$-droop controller starts operating in case the grid voltage exceeds a voltage deviation of $5 \%$ with respect to the nominal voltage. In this way, the delivered active powers of the sources can be changed, 


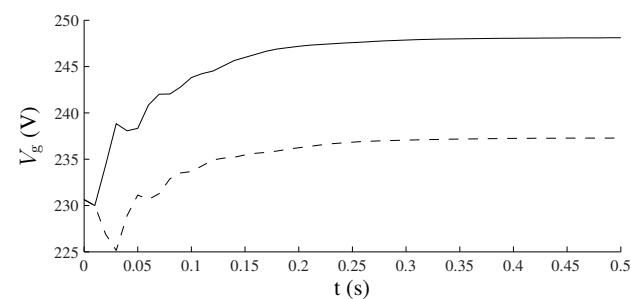

(a) Grid Voltage

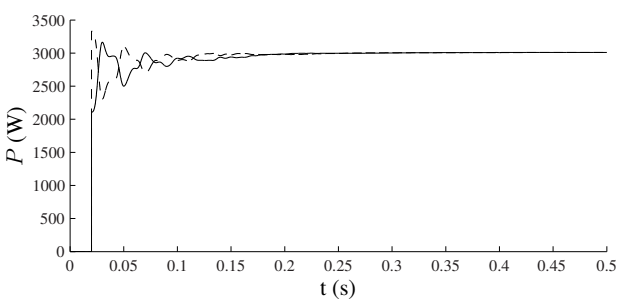

(b) Active Power

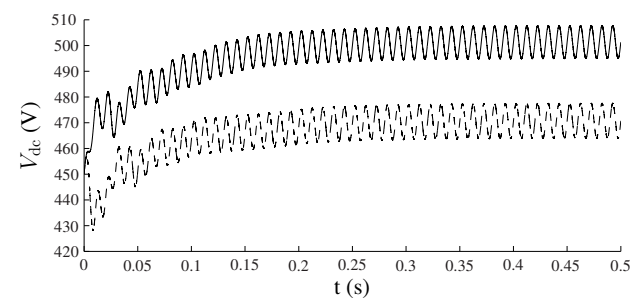

(c) Dc-bus Voltage

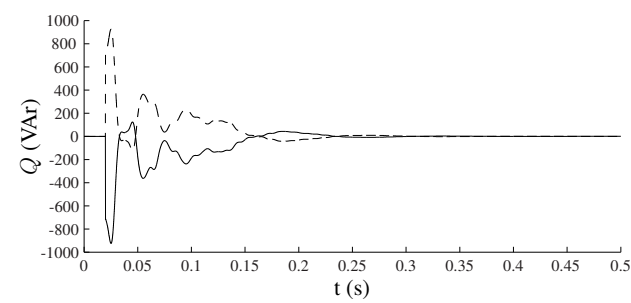

(d) Reactive Power

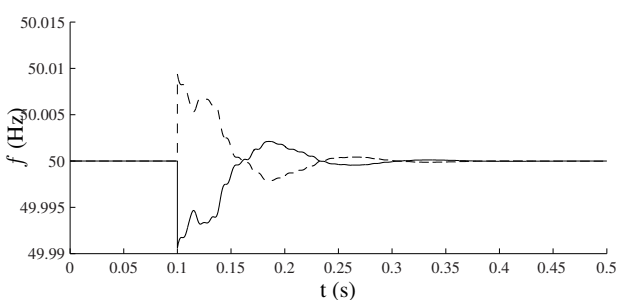

(e) Frequency

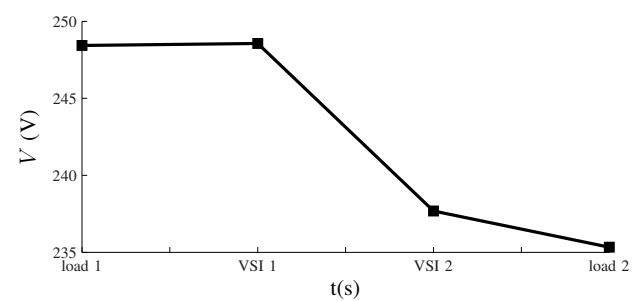

(f) Voltage profile

Fig. 5. Case I: $V_{\mathrm{g}} / V_{\mathrm{dc}}$-droop controller $(-=$ VSI 1 ; --- = VSI 2$)$

avoiding under or over-voltage. As the focus of this paper is on the voltage profile, only the grid voltage, delivered active power and voltage profile are plotted in Fig. 6.

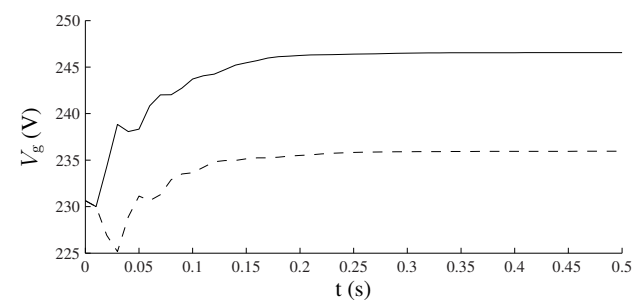

(a) Grid Voltage

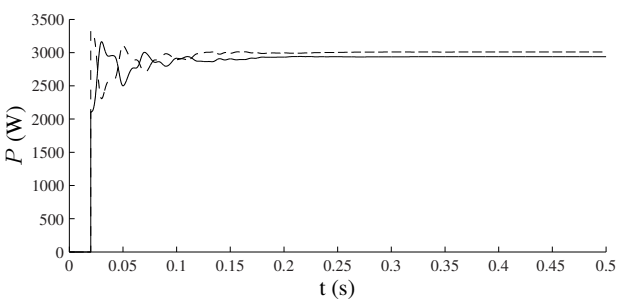

(b) Active Power

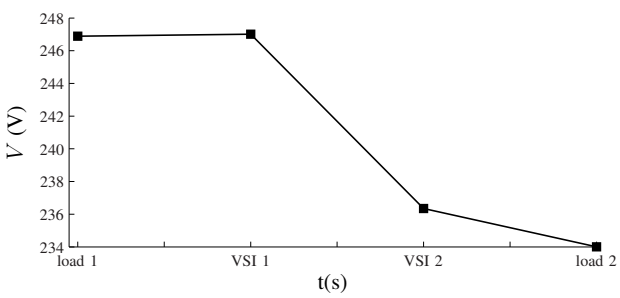

(c) Voltage profile

Fig. 6. Case IIa : $V_{\mathrm{g}} / V_{\mathrm{dc}}$ and $P_{\mathrm{dc}} / V_{\mathrm{g}}$-droop controller $(-=\mathrm{VSI} 1$; --- $=$ VSI 2)

As summarized in Table I, the voltage of both inverters is now decreased and, thus, closer to the nominal voltage of $230 \mathrm{~V}$. In the latter simulation case, the voltage of VSI 2 remained in the $5 \%$ voltage band (lower than $241.5 \mathrm{~V}$ ), thus, $P_{2}$ now remains $3 \mathrm{~kW} . P_{1}$, on the other hand, has decreased to $2939 \mathrm{~W}$ to lower the terminal voltage, because $V_{\mathrm{g}, 1}$ was larger than $1.05 V_{\mathrm{g}, \mathrm{nom}}$.

With the combination of the $V_{\mathrm{g}} / V_{\mathrm{dc}}$-droop controller and the $P_{\mathrm{dc}} / V_{\mathrm{g}}$-droop controller, the advantages of both are exploited. With the $V_{\mathrm{g}} / V_{\mathrm{dc}}$-droop controller, frequent changes of the generated power are avoided, while by including the $P_{\mathrm{dc}} / V_{\mathrm{g}}$-droop controller when a voltage band is exceeded, the microgrid flexibility is higher. Furthermore, the width of constant-power band can be adjusted depending on the nature of the generator. In this way, a deviation of the nominal voltage (in islanded microgrid upto $10 \%$ is tolerable) is used effectively and a distinction between the power control capabilities of the different controllers can be made to limit this voltage deviation. This helps the power balancing, but also makes optimal use of uncontrollable power sources.

2) Case IIb: $0 \%$ constant-power band

In this simulation, only the $P_{\mathrm{dc}} / V_{\mathrm{g}}$-droop controller is used to change the delivered power of the generators for the power sharing and balancing in the grid. The simulation results are depicted in Fig. 7.

As summarized in Table I, the voltages of both inverters now lay even closer to the nominal voltage of $230 \mathrm{~V}$. To decrease the grid voltage, the generated active power of both 


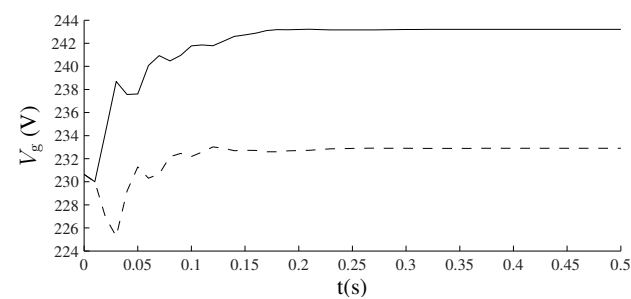

(a) Grid Voltage

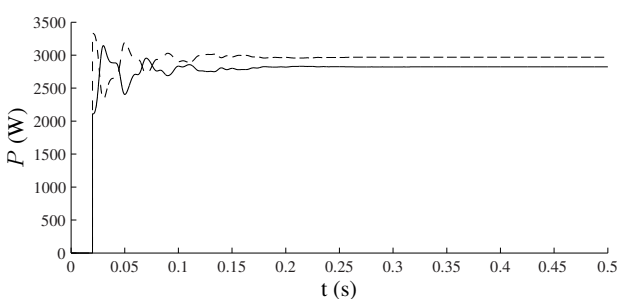

(b) Active Power

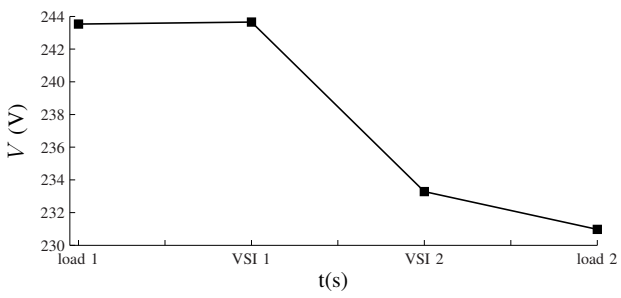

(c) Voltage profile

Fig. 7. Case IIb : $P_{\mathrm{dc}} / V_{\mathrm{g}}$-droop controller (- $=$ VSI 1 ; --- = VSI 2 )

inverters is now lowered to $P_{1}=2923 \mathrm{~W}$ and $P_{2}=2968 \mathrm{~W}$. A disadvantage is that with $P_{\mathrm{dc}} / V_{\mathrm{g}}$-droop control only, the tolerable voltage band is not used effectively, enforcing more frequent changes of the delivered power or more burdening the storage elements; and forcing an operating condition different from the nominal, optimal output power. Therefore, the combination of the $V_{\mathrm{g}} / V_{\mathrm{dc}}$ and $P_{\mathrm{dc}} / V_{\mathrm{g}}$-droop control strategies gives better results, certainly if the constant-power bands are adjusted depending on the characteristics of the power sources.

\section{3) Case IIc: effect of the droops}

In the following simulation, the effect of the droop slopes is studied. Only the $P_{\mathrm{dc}} / V_{\mathrm{g}}$-droop controller is used, and now, the droop slope of VSI 1 becomes $-50 \mathrm{~W} / \mathrm{V}$, increasing the rate of power change of this inverter. Hereby, the delivered active power changes further and the voltage profile is forced closer to the nominal voltage of $230 \mathrm{~V}$.

The simulation results are depicted in Fig. 8. The delivered active powers become $P_{1}=2496 \mathrm{~W}$ and $P_{2}=3037 \mathrm{~W}$. The power $P_{2}$ is now increased as $V_{\mathrm{g}, 2}<V_{\mathrm{g}, \text { nom }}=230 \mathrm{~V}$.

An advantage of the increased droop is that the voltage profile becomes closer to the nominal profile. However, the delivered active powers lay further from the nominal ones. Also, the power source with the highest absolute value of droop, will take a larger part in the power sharing, changing its output power with a larger amount.

4) Case IId: effect of very high droops

In this simulation, only the $P_{\mathrm{dc}} / V_{\mathrm{g}}$-droop controller is used,

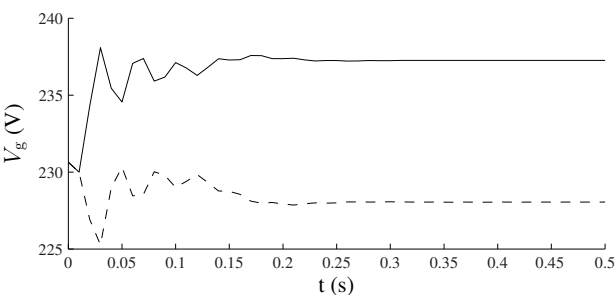

(a) Grid Voltage

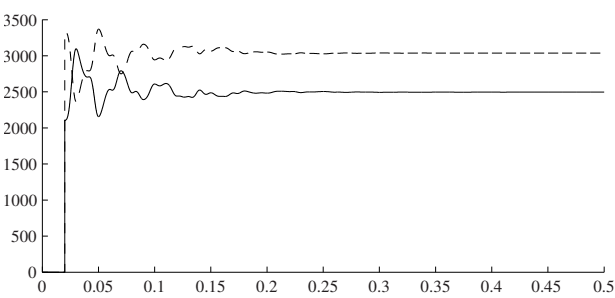

(b) Active Power

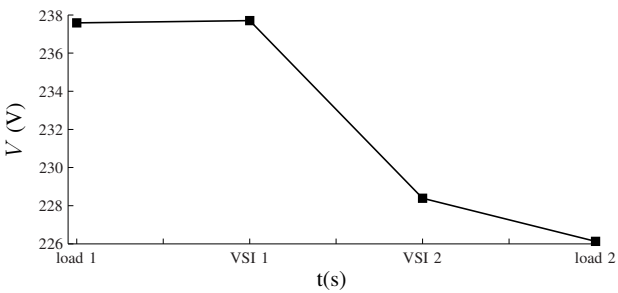

(c) Voltage profile

Fig. 8. Case IIc : $P_{\mathrm{dc}} / V_{\mathrm{g}}$-droop controller with different slopes $(-=$ VSI 1 ; $---=$ VSI 2)

with droops of $-100 \mathrm{~W} / \mathrm{V}$ for both power sources. The simulation results are depicted in Fig. 9. The controllers act more dynamically, closer to the stability margins and the transients have a longer duration. As summarized in Table I, the voltages of both inverters now lay closer to the $230 \mathrm{~V}$ nominal voltage and the generated active power is even more changed (about $2200 \mathrm{~W}$ for the first power source and about $3300 \mathrm{~W}$ for the second one).

Increasing the droops gives better voltage control, but the droop increase is restricted by the stability margins and the acceptable transients.

\section{Case III: constant terminal voltage}

In the following simulation, the generators adjust their power in order to maintain $230 \mathrm{~V}$ terminal voltage. To emulate this behavior, the power source is changed into a constantvoltage source, without restrictions on the delivered power. Thus, the power sharing is not determined by the ratings of the generators, but by the microgrid configuration. The simulation results in Fig. 10 show that $P_{1}$ becomes very small, about $300 \mathrm{~W}$, while $P_{2}$ increases to $5300 \mathrm{~W}$. This control strategy therefore forms an unrealistic scenario as VSI 2 delivers significantly more power than VSI 1.

Therefore, it is concluded that strictly forcing the grid voltage to a predefined value is not an option as it does not take into account the specific ratings of the power sources. Therefore, the combination of the two droop controllers gives good perspectives on the voltage profile in the microgrid. This simulation also shows that the secondary control algorithm 


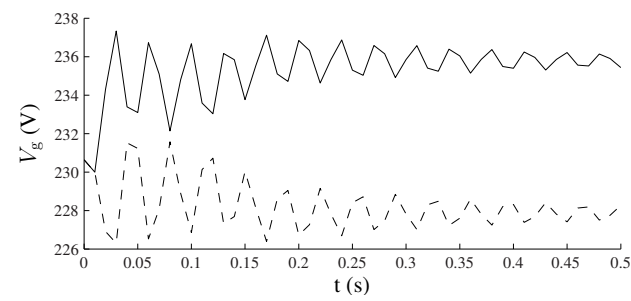

(a) Grid Voltage

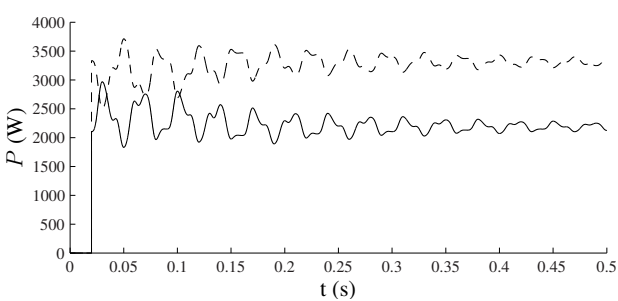

(b) Active Power

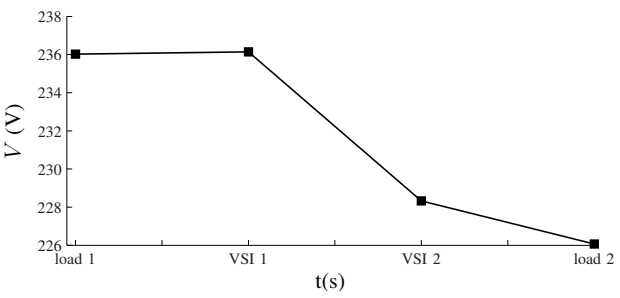

(c) Voltage profile

Fig. 9. Case IId : $P_{\mathrm{dc}} / V_{\mathrm{g}}$-droop controller with high droop slopes $(-=$ VSI $1 ;---=$ VSI 2)

TABLE I

SUMMARY: VOLTAGE PROFILE

\begin{tabular}{|c||c|c|c|c|}
\hline & $V_{1,1}(\mathrm{~V})$ & $V_{\mathrm{g}, 1}(\mathrm{~V})$ & $V_{\mathrm{g}, 2}(\mathrm{~V})$ & $V_{1,2}(\mathrm{~V})$ \\
\hline \hline Case I & 248.42 & 248.56 & 237.69 & 235.33 \\
Case IIa & 246.89 & 247.01 & 236.35 & 234.01 \\
Case IIb & 243.50 & 243.65 & 233.28 & 230.98 \\
Case IIc & 237.59 & 237.71 & 228.39 & 226.13 \\
Case IId & 236.02 & 236.13 & 228.32 & 226.063 \\
\hline
\end{tabular}

may not fix the grid voltage strictly, but still some voltage deviation needs to be tolerated.

\section{CASE STUDY: SECONDARY GOSSIPING CONTROLLERS}

In this case, the gossiping based secondary control strategy of $\S I I-D$ is studied. In this control strategy, a distributed communication algorithm modifies the grid voltage profile, by adjusting the settings of the generators.

The experimental results presented in this section are obtained using a lab-scale low-voltage microgrid with four parallel inverters representing distributed energy sources. During this experiment, the active power load connected to the grid is changed at certain instants, as depicted in the second graph of Fig. 11. The top graph shows the active output power of each inverter, where inverter I and II; and, III and IV, have the same marginal energy production cost curve, respectively. The marginal costs of the various inverters have to become equal for the system to operate in an economically optimal state (tertiary control). As illustrated in the lower graph, this is accomplished most of the time, unless technical issues

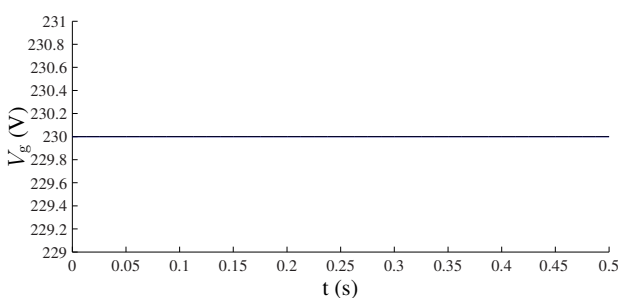

(a) Grid Voltage

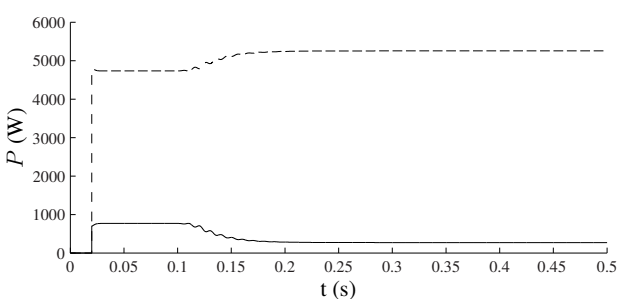

(b) Active Power

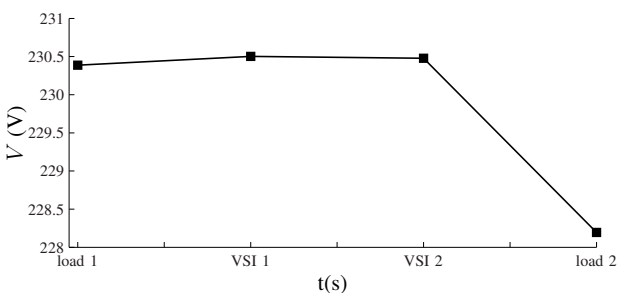

(c) Voltage profile

Fig. 10. Case III : Variable output power, constant terminal voltage $(-=$ VSI $1 ;---=$ VSI 2)

render equation of all marginal costs impossible. For instance, between $\mathrm{t}=300 \mathrm{~s}$ and $500 \mathrm{~s}$, the voltage drop across the line is such that with equal marginal costs, the voltage magnitude is outside the $1 \%$ tolerance for some inverters. In that case, the primary control overrides the tertiary control. As this is no longer an economically optimal state of operation, the cost difference can be considered to be the cost for maintaining adequate voltage quality. Similarly, from $t=500 \mathrm{~s}$ till 700 $\mathrm{s}$, the system load is very low. Inverters I and II are the most expensive units and their output power is reduced to zero, which also causes unequal marginal costs in steadystate. The third graph from the top illustrates the operation of the secondary control. The tolerance band for the voltage magnitude is very small, i.e. $1 \%$. It can be seen that the control is able to control the local voltage magnitudes to be inside the tolerance band in steady-state, with a relatively small deviation outside of this zone immediately after the active power load steps. More details regarding this experiment, as well as results for similar tests are given in [12].

\section{CONCLUSIONS}

In this paper, the voltage profile in an islanded microgrid is studied for different control strategies. In the simulation case I, it is shown that the $V_{\mathrm{g}} / V_{\mathrm{dc}}$-droop control strategy can be used to avoid frequent changes of the generated power, for example in uncontrollable power sources. In microgrids, the voltage can vary within certain limits and by using this control strategy, this tolerable voltage band is used effectively. However, more power flexibility in the islanded microgrid is required to force 

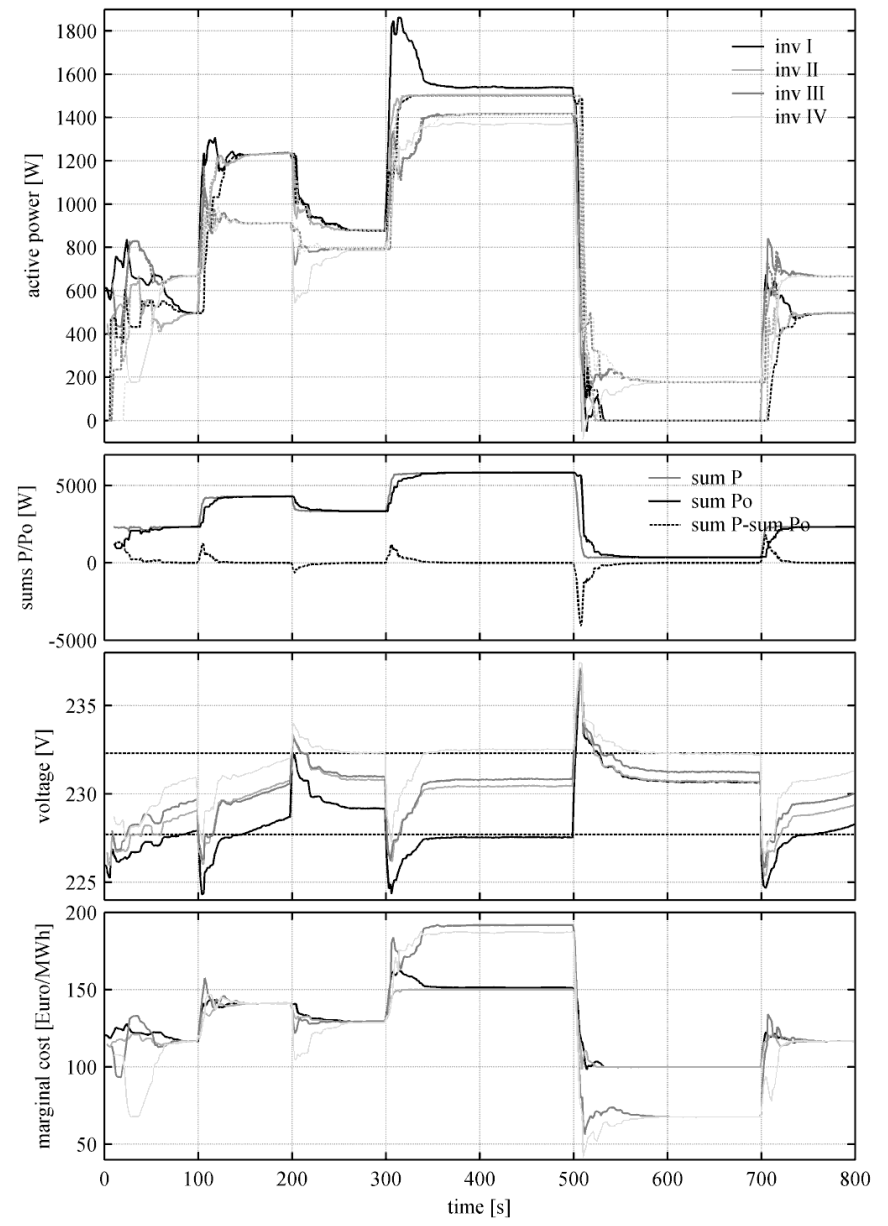

Fig. 11. Experiment with active power load variations (from top to bottom): inverter active output power; load power and total inverter output power; inverter local output voltage magnitude; inverter marginal energy production cost

the grid voltage in the voltage limits. Therefore, in case II, the $V_{\mathrm{g}} / V_{\mathrm{dc}}$-droop control is complemented with $P_{\mathrm{dc}} / V_{\mathrm{g}}$-droop control to change the generated power in case a certain voltage is exceeded. The constant-power band can be adjusted to make a distinction between the sources in highly controllable and less or non-controllable sources. In case II, also the influence of the width of the constant-power band and the droop slopes is studied. It is shown that with a narrowed band, the power changes more and more frequently to keep the grid voltage closer to the nominal voltage. The droop slopes can be adjusted to keep the voltage more closely to its nominal value, but, high droop slopes can give a larger settling time and a more dynamical operation. In case III, the situation is studied where the voltage is forced to the nominal voltage, and now, the delivered power does not depend on the ratings of the sources, but on the microgrid configuration, which should be avoided. Also, stability problems can occur in case the inverter can not change its output power sufficiently. Overall, it is shown that a wisely chosen combination of $V_{\mathrm{g}} / V_{\mathrm{dc}}$ and $P_{\mathrm{dc}} / V_{\mathrm{g}}$-droop control with different constant-power bands, gives a good microgrid operation. Finally, a secondary control based on inter-unit communication in a gossiping algorithm is included to force the voltage in the voltage limits. This control is based on two levels of control: a primary control action for the balancing and a secondary control action for the voltage regulation.

In this paper, it is shown that in an islanded microgrid, the voltage can be determined by a primary, non-communication based control. Also, a secondary control can be included to control the voltage even more strictly if necessary.

\section{ACKNOWLEDGMENT}

This work is financially supported by the FWOVlaanderen (Research Foundation Flanders, Belgium, FWOproject G.0587.07N). The work of T.L. Vandoorn is supported by a Ph.D. fellowship from the FWO-Vlaanderen. The research was carried out in the frame of the inter-university Attraction poles IAP-VI-021, funded by the Belgian Government.

\section{REFERENCES}

[1] D. Zhu, R. P. Broadwater, T. Kwa-Sur, R. Seguin, and H. Asgeirsson, "Impact of dg placement on reliability and efficiency with time-varying loads," IEEE Trans. Power Syst., vol. 21, no. 1, pp. 419-427, Jan. 2006.

[2] D. T. Wang, L. F. Ochoa, and G. P. Harrison, "Dg impact on investment deferral: Network planning and security of supply," IEEE Trans. Power Syst., vol. 25, no. 2, pp. 1134-1141, May 2010.

[3] T. E. McDermott and R. C. Dugan, "PQ, reliability and DG," IEEE Ind. Appl. Magazine, vol. 9, no. 5, pp. 17-23, Sept. 2003.

[4] J. Driesen and F. Katiraei, "Design for distributed energy resources: Microgrid planning and architectures for improved reliability and integration," IEEE Power \& Energy Magazine, vol. 6, no. 3, pp. 30-40, May 2008.

[5] A. A. Salam, A. Mohamed, and M. A. Hannan, "Technical challenges on microgrids," ARPN Journal of Engineering and Applied Sciences, vol. 3, no. 6, pp. 64-69, Dec. 2008.

[6] C. M. Colson and M. H. Nehrir, "A review of challenges to real-time power management of microgrids," in Proc. of the 2009 IEEE Power \& Energy Society (PES) General Meeting, Calgary, Alberta, Canada, July 26-30,

[7] T. L. Vandoorn, B. Renders, B. Meersman, L. Degroote, and L. Vandevelde, "Power balancing in islanded microgrids by using a dc-bus voltage reference," in 20th Internat. Symp. on Power Electronics, Electrical Drives, Automation and Motion (SPEEDAM 2010), Pisa, Italy, June 14 $16,2010$.

[8] A. Engler, "Applicability of droops in low voltage grids," DER journal, no. 1, Jan. 2005.

[9] T. L. Vandoorn, B. Renders, B. Meersman, L. Degroote, and L. Vandevelde, "Reactive power sharing in an islanded microgrid," in 45th International Universities Power Engineering Conference (UPEC 2010), Cardiff, Wales, Aug. 31 - Sep. 3, 2010.

[10] K. De Brabandere, K. Vanthournout, J. Driesen, G. Deconinck, and R. Belmans, "Control of microgrids," in Proceedings of the IEEE Power Engineering Society (PES) General Meeting, Tampa, Florida, USA, June 24-28, 2007.

[11] M. Bollen, J. Zhong, O. Samuelsson, and J. Björnstedt, "Performance indicators for microgrids during grid-connected and island operation," in Proc. IEEE Power Tech. Conf., Bucharest, Romania, June 28 - July 2, 2009.

[12] K. De Brabandere, "Voltage and frequency droop control in low voltage grids by distributed generators with inverter front-end," Ph.D. dissertation, KU Leuven, Faculteit Ingenieurswetenschappen, Departement elektrotechniek, afdeling elektrische energie en computerarchitecturen, Kasteelpark Arenberg 10, B-3001 Leuven, België, Oct. 2006.

[13] K. Debrabandere, B. Bolsens, J. Van den Keybus, A. Woyte, J. Driesen, and R. Belmans, "A voltage and frequency droop control method for parallel inverters," IEEE Trans. Power Electron., vol. 22, no. 4, pp. 1107-1115, July 2007.

[14] UCTE. (2009, Mar. 19,) Operation handbook policy 1: Load-frequency control and performance. [Online]. Available: http://www.entsoe.eu

[15] K. Vanthournout, "A semantic overlay network-based robust datainfrastructure applied to the electric power grid," $\mathrm{Ph} . \mathrm{D}$. dissertation, Catholic University of Leuven, Belgium, FFaculty of Engineering Sciences, July 2006. 